\title{
Implantation of a new enteral stent in obstructive colorectal cancer using interventional radiology in patients over 70 years of age
}

\author{
Tomasz Miłek, Piotr Ciostek \\ Department of General and Vascular Surgery, Medical University of Warsaw, Warsaw, Poland
}

Videosurgery Miniinv 2015; 10 (2): 155-160

DOI: $10.5114 /$ wiitm.2015.52266

\begin{abstract}
Introduction: Colorectal cancer is the second leading cause of death due to cancer in Poland. The traditional approach to treat patients included a surgical procedure. Irrespective of the surgical method being used, surgical treatment of malignant colorectal obstruction is associated with prolonged hospitalisation, and the postoperative mortality rate is approximately 5-11\%. Due to these problems, more interest has been shown in less invasive methods. Prosthesis implantation is a leading endoscopic method used currently in palliative or preoperative treatment.

Aim: To compare the results of implantation of traditional stents with the results of implantation of an own stent using minimally invasive methods.

Material and methods: Left-sided colon obstruction due to cancer was an indication for transplantation. All patients were aged over 70 years and had serious concomitant diseases. The control group included 50 patients with colorectal cancer who received traditional stents in the period 2009-2011. Our stent covers only the internal length of a tumour. It is not equipped with anti-migration flares. To minimize the risk of migration it has a system of hooks that are responsible for permanent anchorage of the stent within the tumour mass.

Results: Implantation technical and clinical success defined as effective decompression of intestinal obstruction was $100 \%$ in both groups. There were 2 cases of stent migration in the control group.

Conclusions: It is possible to achieve a secure surgical anastomosis after intestinal decompression. Stent implantation is fast and safe thanks to the positioning system that was used. The use of labelled hooks is a secure anti-migration solution.
\end{abstract}

Key words: colorectal cancer, endoscopic surgery, stent.

\section{Introduction}

Colorectal cancer (CRC) is the second cause of death due to cancer in Poland. According to estimates, 15,000 new cases and 11,000 deaths are observed in both sexes every year. Many patients do not have symptoms at diagnosis; however, it is estimated that $7-29 \%$ report with colorectal obstruction or it develops in the course of disease. Malignancies account for approximately $85 \%$ of causes of colorectal obstruction, and they usually indicate locally and systemically advanced disease. The traditional approach to treat such patients included a surgical procedure with resection and a primary anastomosis or a two-stage procedure involving initial resection followed by final colostomy (Hartmann's procedure) and subsequent restoration of the gastrointestinal tract continuity. However, both approaches are associated with several limitations: a single procedure is more difficult from a technical point of view or impossible due to intestinal wall damage, and only $40 \%$ of patients with malignant left-sided colon ob-

\section{Address for correspondence}

Tomasz Miłek MD, Department of General and Vascular Surgery, Medical University of Warsaw, 8 Kondratowicza St, 03-242 Warsaw,

Poland, phone: +48 604422 345, e-mail: tomasz_milek@wp.pl 
struction are eligible for such a procedure. Usually, when left-sided colon obstruction is present, it is necessary to perform a two-stage procedure, and patients in this group are often elderly, with many concomitant diseases and with more advanced disease, and therefore with a higher risk of postoperative complications. As a result, it is often not possible to perform a second procedure to close the fistula; hence permanent colostomy is present and it negatively affects the quality of life.

Irrespective of the surgical method being used, surgical treatment of malignant colorectal obstruction is associated with prolonged hospitalisation, and the postoperative mortality rate is approximately $5-11 \%$. Due to these problems, more and more interest has been shown in more modern, less invasive alternative methods and methods preceding surgical treatment: prosthesis implantation, laser ablation, argon plasma coagulation, endoscopic dilation, cryotherapy, electrocauterisation and endoscopic administration of agents resulting in tumour necrosis. Based on these comments, prosthesis implantation seems to be a leading endoscopic method used currently in palliative or preoperative treatment.

In 1990 Dohmoto was the first to publish a description of enteral stent implantation. He used a metal endoprosthesis in order to restore intestinal patency in a patient with unresectable colorectal cancer. In 1994 Tejero et al. presented a case when an enteral stent was used in a patient with resectable colorectal cancer complicated by obstruction. It was the first time that a stent was used to decompress the intestines and to prepare a patient for resection [1-4].

Between 1990 and 2012, more than 65 studies on the results of metal prosthesis implantation in palliative treatment and as a graft for surgical treatment of colorectal cancer were published.

Construction of the stent was preceded by several years of experience in stent implantation and constructions from various companies. From the very beginning, we used stents from the Czech company ELLA, including the Danis stent to control haemorrhage from oesophageal varices. In our opinion, the balloon positioning system used in these stents is based on the idea of the usage of the Sengstaken-Blakemore tube. In our stent the main idea of using the balloon for accurate positioning of stenosis was its good visibility on X-ray, which is missing in the Danis stent. The use of the second channel to provide a contrast agent outside the balloon allows one to specify the position of the set. This ensures safety during implantation as perforation of the gastrointestinal tract or abnormal position of the intestine after previous operations can be excluded. The Danis stent does not have such solutions and thus may result in incorrect stent positioning.

As a result of advances in the field of endoscopic methods and technological progress with regard to construction of new materials, it was possible to use nitinol stents. Nitinol is an alloy of nickel and titani$\mathrm{um}$, and it is characterised by excellent elasticity and autonomous memory of its primary shape.

\section{Aim}

The study aimed to compare the results of implantation of traditional stents with results of implantation of an own stent using minimally invasive methods.

\section{Material and methods}

Traditional enteral stents have been used since 2009. Based on our own experience regarding stent implantation due to malignant colorectal obstruction, a decision was made to manufacture a self-constructed enteral stent. Work started in 2010, and Balton was the company that manufactured the first Polish enteral stent. Designs of stents by leading global manufacturers were considered when a self-constructed stent was designed. The rule was to use the best materials and to eliminate defects of stents available on the market. Implantation of a self-constructed expandable stent was performed in 45 subjects. Forty-five stents with the diameter of $25 \mathrm{~mm}$ and length of $60-80 \mathrm{~mm}$ were implanted in the period 2012-2014.

Left-sided colon obstruction due to cancer was an indication for transplantation. All patients were over 70 years old and had serious concomitant diseases. The Bioethics Committee at the Medical University of Warsaw approved the stent usage. A control group included 50 patients with colorectal cancer who received traditional stents in the period 2009-2011. In both groups uncoated stents were used.

Tumour stenosis consists of three sections: the critical stenosis, the tumour mass narrowing above and below the obstruction, and the obstruction caused by swelling of the intestinal wall located proximally and distally to the tumour. 
Based on the numerous computed tomography studies it is considered that to cover the entire length of the stenosis, stents of two dimensions are needed: $6 \mathrm{~cm}$ and $8 \mathrm{~cm}$. By covering the entire length of the stenosis and obstruction of the tumour it prevents restenosis.

Our stent covers only the internal length of a tumour. It is an innovatory approach, as traditional enteral stents have, apart from the main tube used to expand the tumour, two flares, distal and proximal, that exceed the tumour lumen significantly. They function as elements preventing stent migration. They expand in the lumen of the healthy intestine and a priori irritate and damage the distal mucosa and the mucosa of the intestine above the stenosis, after evacuation.

For that reason, our stent is not equipped with anti-migration flares. However, there is consequently a higher risk of stent migration. For this reason we developed our own, first ever, system of hooks that are responsible for permanent anchorage of the stent within a tumour mass (Photo 1). They are located in the centre, opposite to one another. The length of the hooks was designed so that there would not be a risk of intestinal perforation but at the same time the hooks would secure the stent at the site of the obstruction safely. According to an intraoperative assessment of the intestinal wall thickness performed in 50 patients, the minimum intestinal thickness was between $5 \mathrm{~mm}$ and $4.7 \mathrm{~cm}$ at the site of a tumour. Hooks of a self-constructed stent were $3 \mathrm{~mm}$ long. It is a safe length so perforation is not possible, and it is also sufficient to anchor the stent securely in the intestinal wall.

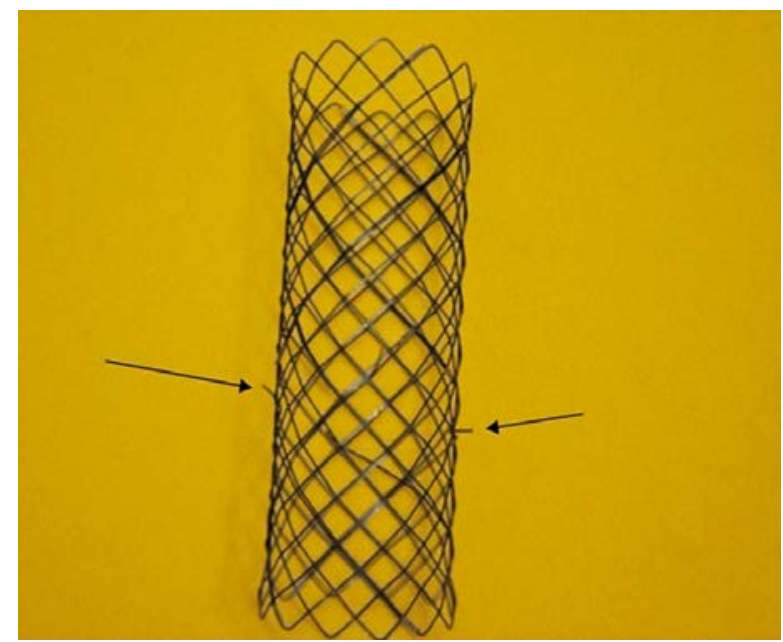

Photo 1. Hooks
In order to deploy the stent safely at the site of obstruction, it has to be well visible under X-ray monitoring. Tests of stent visibility were conducted and X-ray scans of various braided stent designs were taken. As stent implantation in the intestinal lumen is a dynamic procedure that constantly changes along with intestinal peristalsis and respiratory movements, stent visibility was enhanced by placing distal and proximal markers of higher radiopacity. Additionally, at the site of anti-migration hooks, markers located in the central part of the stent were placed in order to facilitate their precise deployment in a tumour mass (Photo 2).

A possibility of precise stent implantation at the site of obstruction constitutes an original modification in the stent design. It is possible thanks to the design of the distal part of the delivery system with the stent. The system ends with a $5-\mathrm{mm}$ bulb tip that enables free and atraumatic passage through the obstruction site under X-ray monitoring (Photo 3). $X$-ray monitoring was used to identify stenosis for stent implantation in low-lying tumours, while endoscopy was used in high-lying tumours. Savary-Gilliard dilators were used only in case of ability to pass the stenosis. In those cases, the expansion was continued only until the set could be placed safety.

At its base there is a folded stent, and inside there is a catheter ending with a balloon. The balloon is hidden at the base of the bulb tip while being deployed. When the stent passes through the obstruction, the balloon is filled with a radiopaque contrast agent that is visible under $\mathrm{X}$-ray monitoring during an examination. The moment when the stent passes through a stenosis is also visible, as a con-

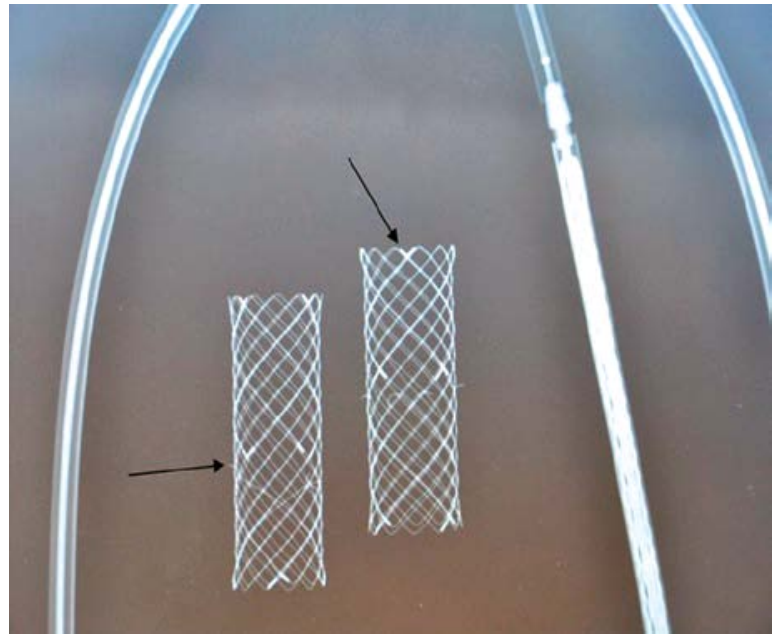

Photo 2. Marker 


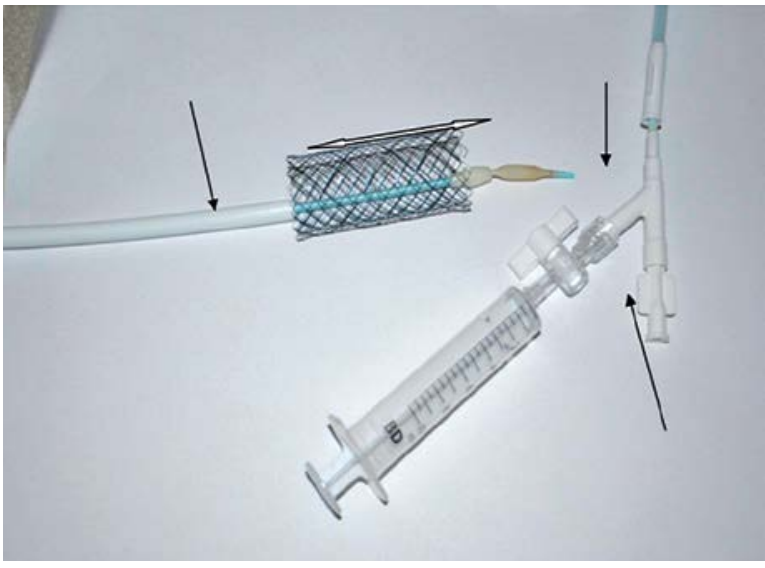

Photo 3. System ends

trast agent is administered into the system and it spreads on its upper surface when it has passed a critically narrowed tumour segment. The base of an expanded balloon is located in the direct vicinity of the stent (Photo 4). When the system is withdrawn, the balloon base is supported on the initial segment of a malignant stenosis. At this moment we start to release the stent located below. The stent length is selected according to stenosis length assessed based on a computed tomography scan performed prior to the procedure.

As a result of positioning a cancer stenosis site with a balloon filled with a contrast agent, it is possible to precisely determine a site of a cancer stenosis and to deploy the stent covering only the internal surface of the tumour. When the stent has been released, the balloon with contrast agent is emptied and removed along with the system under radiologic supervision. However, a contrast agent is administered earlier in order to check stent expansion.

\section{Results}

Stents were applied in palliative treatment, obstructive colorectal cancer carcinoma and as a stage procedure prior to scheduled surgery. Implantation technical and clinical success defined as effective decompression of intestinal obstruction was $100 \%$ in both groups. There were 2 cases of stent migration in the control group. In both cases the stent location changed due to reduced tumour mass and intestinal inflammation following chemotherapy. There were also two cases of intestinal perforation in the control group. In both cases perforation was observed 27 and 35 days after implantation. During

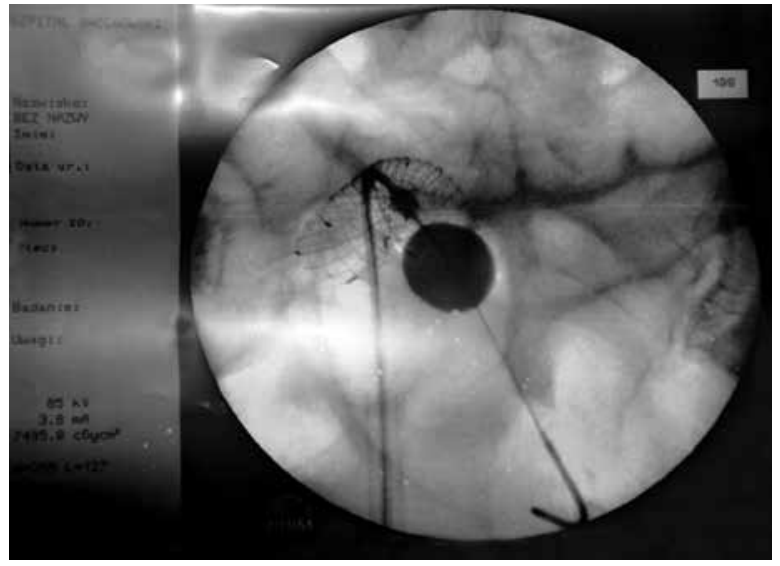

Photo 4. Release the stent located below balloon

surgery the perforation in both cases developed at the site where the intestine was constantly irritated by the upper flare. In our opinion, this type of complication is strongly affected by the intestinal flexure above the tumour due to adhesions associated with previous operations (Table I).

Resection surgery after intestinal decompression was performed in $67 \%$ in the study group and in $76 \%$ in the control group. A primary anastomosis was performed in all patients considered eligible for surgery. In $15 \%$ of patients in the control group jejunostomy was created in order to decompress a primary anastomosis. It included patients with rectal cancer, and the cause of decompressing jejunostomy included inflammation of the rectal mucosa due to irritation caused by a lower stent flare and fear of leakage at the anastomosis site (Table II).

\section{Discussion}

Recently, efforts have been made to avoid emergency CRC surgery. There have been attempts of colonoscopic intestinal decompression in cases of obstruction, and in some centres self-expandable stents are implanted in the intestine at the site of stenosis. As a result, it is possible to decompress the intestine and to gradually prepare a patient for a scheduled procedure, increasing its chances of success [5-7]. In the case of a serious general condition or advanced cancer, stent implantation may be good and sufficient palliative treatment. According to reports, stents in such cases were present in the intestine for 2 to 64 weeks [8, 9]. Martinez-Santos et al. compared emergency surgical treatment with intestinal decompression with stents in patients with 
Table I. Indication - palliative and bridge to surgery and complications

\begin{tabular}{|lcccccc|}
\hline Group & $\begin{array}{c}\text { Surgical } \\
\text { treatment } \\
n(\%)\end{array}$ & $\begin{array}{c}\text { Palliative } \\
\text { treatment } \\
n(\%)\end{array}$ & $\begin{array}{c}\text { Technical } \\
\text { success } \\
n(\%)\end{array}$ & $\begin{array}{c}\text { Stent } \\
\text { migration } \\
n(\%)\end{array}$ & $\begin{array}{c}\text { Re-obstruction } \\
n(\%)\end{array}$ & $\begin{array}{c}\text { Perforation } \\
n(\%)\end{array}$ \\
\hline $\begin{array}{l}\text { Study } \\
(N=45)\end{array}$ & $30(67)$ & $15(33)$ & $45(100)$ & 0 & 0 & 0 \\
\hline $\begin{array}{l}\text { Control } \\
(N=50)\end{array}$ & $38(76)$ & $12(24)$ & $50(100)$ & $2(4)$ & 0 & $2(4)$ \\
\hline
\end{tabular}

left-sided colorectal obstruction due to cancer. Stents were used in order to delay surgery or as palliative treatment. They found that symptoms of obstruction regressed in $95 \%$ of cases after 8 to $96 \mathrm{~h}, 36.4 \mathrm{~h}$ on average. As a result, in patients in whom it was possible to wait and perform surgery as scheduled, intestinal resection and a primary anastomosis were performed statistically significantly more often $(p<0.0025)$ compared to patients operated on as an emergency $(88 \%$ vs. $46 \%$ ), and Hartmann's procedure was performed more rarely ( $p<0.0025)$ (12\% vs. 54\%); additionally, their hospitalisation was shorter, as well as stay at the intensive care unit (ICU), and there were fewer postoperative complications in this group [10].

Surgical treatment for colorectal cancer in the elderly is associated with a mortality rate of $1.0-25 \%$ (Table I) [6, 11-16]. Papers comparing CRC treatment in the elderly and in younger patients reported that the mortality rate in the elderly is statistically significantly higher $[5,15,17]$. The complication rate in this group is significantly increased due to malignant colon obstruction. Therefore, as a result of using stents to decompress the intestine, it is possible to gain more time to prepare a patient, and to improve their clinical status. Surgery performed for malignant colorectal obstruction often has two stages. The first is a life-saving procedure to remove the tumour and create an ostomy. The second stage is performed several months later and it includes restoration of the gastrointestinal tract continuity [12]. Based on our experience, the second stage of surgical treatment is not performed in patients aged over 70 years. Advanced cardiovascular and respiratory disease ASA grade III/IV is usually the most common reason why patients are not considered eligible for surgery $[8,13$, 18-24]. Therefore, good condition of the intestines subject to an anastomosis is a key factor when considering eligibility of patients for surgery. With regard to traditional stents, anti-migration flares reaching far outside the tumour mass are responsible for constant irritation leading to inflammation. Constant irritation
Table II. Jejunostomy to decompress anastomosis

\begin{tabular}{|lcc|}
\hline $\begin{array}{l}\text { Group treated with } \\
\text { surgery }\end{array}$ & $\begin{array}{c}\text { Primary } \\
\text { anastomosis }\end{array}$ & $\begin{array}{c}\text { Jejunostomy } \\
\text { to decompress } \\
\text { anastomosis }\end{array}$ \\
\hline Study group -30 & $100 \%$ & $0 \%$ \\
\hline Control group - 38 & $100 \%$ & $6(15 \%)$ \\
\hline
\end{tabular}

may result in intestinal perforation. In our material this complication was observed in 2 patients in the control group. During an operation we observed perforation at the site where a stent anti-migration flare was in contact with the intestine that was positioned not in an anatomical position due to previous surgery. This complication was not observed in a group with a new stent implanted, and it can be explained by the fact that this stent covers only the tumour mass. Stent migration is the second most common complication associated with implantation. It is present in $1-11 \%$ of cases. Systemic oncological treatment reducing tumour mass and radiation therapy are thought to be the main causes of migration. Other causes of migration might include implantation of coated stents. In our material we observed migration in 2 (4\%) patients in a traditional stent group. Migration was observed after chemotherapy had been completed, 5 and 7 months after implantation. In our opinion, lack of stent migration in patients in whom a self-constructed stent was implanted is associated with the presence of anti-migration hooks that provide secure anchorage for a stent inside a tumour. We believe the lack of complications in the study group is due to experience gained before the new stent was introduced.

Sixty-seven percent of patients in the study group and $76 \%$ of patients in the control group were operated on when the obstruction had regressed and their general condition had improved. A primary anastomosis was performed in all patients in both groups, after an appropriate resection was performed (Table II). Colonoscopy was performed in 
all patients prior to surgery to assess stent location and to evaluate the whole colon. The examination in the control group revealed inflammation of the intestinal mucosa below and above the tumour that was caused by irritation due to anti-migration flares following intestinal decompression. It was not significant in cases where the tumour was located above the rectum, as the resection included the whole pathologically changed intestinal segment. However, if the tumour is located in the rectum, each centimetre of the healthy intestine is valuable in order to create a tight anastomosis. In 6 (15\%) patients in the control group, jejunostomy was created to decompress an anastomosis due to inflammation observed during colonoscopy. The fistula was closed after 4 weeks. The surgery was preceded by colonoscopy of the area of the anastomosis.

\section{Conclusions}

When a stent is implanted in the sigmoid-rectal area, it is possible to achieve a secure surgical anas tomosis after intestinal decompression. Stent implantation is fast and safe thanks to the positioning system that was used. The use of labelled hooks is a secure anti-migration solution.

\section{Conflict of interest}

The authors declare no conflict of interest.

\section{References}

1. Lelcuk S, Ratan J, Klausner JM, et al, Endoscopic decompression of acute colonic obstruction. Avoiding staged surgery. Ann Surg 1986; 203: 292-4.

2. Dohmoto M, Hunerbein M, Schlag PM. Palliative endoscopic therapy of rectal carcinoma. Eur J Cancer 1996; 32A: 25-9.

3. Dohmoto M, Rupp KD, Hohlbach G. Endoscopically implanted prosthesis in rectal carcinoma. Dtsch Med Wochenschr 1990; 115: 915.

4. Tejero E, Mainar A, Fernandez L, et al. New procedure for the treatment of colorectal neoplastic obstructions. Dis Colon Rectum 1994; 37: 1158-9.

5. Saida Y, Sumiyama Y, Nagao J, Takase M. Stent endoprosthesis for obstucting colorectal cancers. Dis Colon Rectum 1996; 39: 552-5.

6. Kamocki ZK, Zaręba KP, Bandurski R, et al. Own experiences of endoscopic self-expandable stent placement for malignant colorectal ileus. Videosurgery Miniinv 2014; 9: 59-63.

7. Miłek T, Ciostek P, Woźniak W, et al. Preliminary results of the use of self-expanding nitinol stents in inoperable gastrointestinal cancers. New Medicine 2011; 15: 79-83.
8. Tsugawa K, Koyanagi N, Hashizume M, et al. Therapeutic strategy of emergency surgery for colon cancer in 71 patients over 70 years of age in Japan. Hepatogastroenterology 2002; 49: 393-8.

9. Akle CA. Endoprosthesis for colonic strictures. Br J Surg 1998; 85: 310-4.

10. Martinez-Santos C, Lobato RF, Fradejas JM, et al. Self-expandable stent before elective surgery vs. emergency surgery for the treatment of malignant colorectal obstructions: comparison of primary anastomosis and morbidity rate. Dis Colon Rectum 2002; 45: 401-6.

11. Lee L, Jannapureddy M, Albo D, et al. Outcomes of Veterans Affairs patients older than age 80 after surgical procedures for colon malignancies. Am J Surg 2007; 194: 646-51.

12. Colorectal Cancer Collaborative Group. Surgery for colorectal cancer in elderly patients: a systematic review. Lancet 2000; 356: 968-74.

13. Spivak H, Maele DV, Friedman I, Nussbaum M. Colorectal surgery in octogenarians. J Am Coll Surg 1996; 183: 46-50.

14. Fabre JM, Rouanet P, Ele N, et al. Colorectal carcinoma in patients aged 75 years and more: factors influencing short and long term operative mortality. Int Surg 1993; 78: 200-3.

15. Agarwal N, Leighton L, Mandile MA, et al. Outcomes of surgery for colorectal cancer in patients age 80 years and older. Am J Gastroenterol 1990; 85: 1096-101.

16. Ong ES, Alassas M, Dunn KB, Rajput A. Colorectal cancer surgery in the elderly: acceptable morbidity? Am I Surg 2008; 195 : 344-8.

17. Marusch F, Koch A, Schmidt $U$, et al. The impact of the risk factor "Age" on the early postoperative results of surgery for colorectal carcinoma and its significance for perioperative management. World J Surg 2005; 29: 1013-22.

18. Lindmark G, Pahlman L, Enblad P, Glimelius B. Surgery for colorectal cancer in elderly patients. Acta Chir Scand 1988; 154: 659-63.

19. Smith JJ, Lee J, Burke C, et al. Major colorectal cancer resection should not be denied to the elderly. EJSO 2002; 28: 661-6.

20. Mulcahy HE, Patchett SE, Daly L, O'Donoghue DP. Prognosis of elderly patients with large bowel cancer. Br J Surg 1994; 81: 736-8.

21. Irvin TT. Prognosis of colorectal cancer in the elderly. $\mathrm{Br}$ J Surg 1988; 75: 419-21.

22. Fitzgerald SD, Longo WE, Daniel GL, Vernava AM. Advanced colorectal neoplasia in the high-risk elderly patient: is surgical resection justified? Dis Colon Rectum 1993; 36: 161-6.

23. Mäkelä JT, Kiviniemi H, Laitinen S. Survival after operations for colorectal cancer in patients aged 75 years or over. Eur J Surg 2000; 166: 473-9.

24. Ozoux JP, de Calan L, Perrier M, et al. Surgery for carcinoma of the colon in people aged 75 years and older. Int J Colorect Dis 1990; 5: 25-30.

Received: 22.01.2015, accepted: 12.05.2015. 